\title{
El rol del lugar y el capital social en la resiliencia comunitaria posdesastre. Aproximaciones mediante un estudio de caso después del terremoto del $27 / \mathrm{F}$
}

Claudia González-Muzzio. Ámbito Consultores Ltda., Santiago, Chile.

RESUMEN | Generar comunidades urbanas resilientes a los desastres es una necesidad, debido a que la mayor parte de la población vive en ciudades y estas son altamente vulnerables a la ocurrencia de tales eventos. Mediante un estudio de caso en el contexto urbano, se analiza el rol del lugar y del capital social en el período de emergencia luego del terremoto del 27 de febrero de 2010 en Chile. Los resultados indican que ambos factores modificaron fuertemente la resiliencia inherente de la ciudad y de su comunidad. Al verse sobrepasada inicialmente la capacidad de absorber los impactos generados por el evento, los habitantes de las zonas afectadas se vieron obligados a adaptarse rápidamente a la nueva situación, aprovechando para ello los recursos disponibles en el área. El surgimiento de nuevos grupos y comportamiento emergentes, así como las características del lugar, contribuyeron positivamente a mejorar la resiliencia adaptativa de la comunidad. Estos factores deben considerarse en la planificación y/o reconstrucción de ciudades con mayores niveles de resiliencia.

PALABRAS CLAVE | capital social, planificación urbana, vulnerabilidad.

ABSTRACT | Generating urban communities resilient to disasters is a necessity, because most of the population lives in cities, which are highly vulnerable to disasters. By means of a case study in an urban context, the role of place and social capital is analyzed during the emergency period following the earthquake occurred on 27 February 2010 in Chile. Results indicate that both factors strongly modified the inherent resilience of the city and the community. At first, citizens' capacity to absorb shocks generated b the event was surpassed, forcing people to adapt quickly to the new situation, drawing on the resources available in the area. The emergence of groups and unheard of behavior, as well as site characteristics, contributed positively to improve the adaptive resilience of the community. These factors should be considered in planning and/or reconstruction of more resilient cities.

KEY WORDS | social capital, urban planning, vulnerability.

Recibido el 15 de marzo de 2011, aprobado el 27 de septiembre 2012

E-mail: Claudia González-Muzzio, ccgonzalezm@gmail.com 


\section{Introducción}

A pesar de los esfuerzos hechos en todo el mundo a partir de la década de los noventa para disminuir la vulnerabilidad a los desastres y las pérdidas humanas y materiales causadas por estos, la población afectada por desastres "naturales" ha ido en aumento, así como los costos económicos de los mismos. Desde el año 2000 en adelante, más de 2,7 billones de personas se han visto afectadas, las pérdidas económicas alcanzaron 1,3 trillón de dólares y murió 1,1 millón de personas (The United Nations Office for Disaster Risk Reduction [UNISDR], 2012). El año 2010, el terremoto de Haití fue el más mortífero, con más de 220.000 víctimas fatales, mientras el terremoto de Chile fue el más costoso, con pérdidas que alcanzaron los 30.000 millones de dólares. Y en el terremoto de Japón en 2011 murieron casi 20.000 personas y se registraron daños por 210.000 millones de dólares (EM-DAT, 2012).

El aumento de la población vulnerable a los desastres no se debe solo al crecimiento demográfico, sino a que la gente se concentra mayoritariamente en áreas urbanas. Más del $50 \%$ de la población mundial vive en ciudades y, en Chile, se estimaba que - al año 2010- lo hacía el $87 \%$ de la población (Instituto Nacional de Estadísticas [INE], 2005), principalmente en las áreas centro y sur del país. Por ello, si bien la reconstrucción sustentable de las áreas dañadas tras un desastre es un imperativo, es necesario considerar que la mayoría de la población chilena es vulnerable a la ocurrencia de desastres gatillados por eventos naturales. Es preciso pensar en cómo reducir la vulnerabilidad de las áreas urbanas, no solo cuando han sido afectadas por un evento, sino con anterioridad a que ello ocurra. El saber que "somos un país sísmico" o "permanentemente expuesto a los desastres naturales", frases recurrentes en estos días, no basta.

Mediante el análisis de lo ocurrido en una comunidad urbana después del terremoto de 2010, se busca identificar cuál fue el rol del lugar y del capital social en la resiliencia comunitaria posdesastre durante el primer mes que siguió al evento, y dar cuenta de la interacción existente entre ambos factores.

\section{Contexto}

El terremoto del 27 de febrero de 2010 en Chile fue percibido por alrededor del $80 \%$ de la población del país y afectó a aproximadamente a 2,5 millones de personas. La magnitud registrada de $8,8 \mathrm{M}_{\mathrm{w}}$ (magnitud de momento sísmico) lo convierte en el sexto terremoto más grande del cual se tenga registro. Más de novecientas ciudades y localidades menores fueron afectadas, cinco de ellas con más de 100.000 habitantes y cuarenta y cinco de más de 5.000 (Ministerio de Vivienda y Urbanismo [Minvu], 2010).

La región del Biobío fue una de las que sufrió más daños, por lo que, en conjunto con otras tres, fue declarada zona de catástrofe tras el sismo. El colapsado edificio "Alto Río”, en Concepción, se convirtió en símbolo de la tragedia a nivel nacional, mientras que el tsunami que siguió al terremoto afectó severamente a Talcahuano y San Vicente, una de las principales áreas portuarias del país, y también 
a importantes industrias y algunos poblados costeros de la zona, como Dichato. Al igual que en otras áreas, los sistemas de comunicaciones colapsaron y los servicios básicos se vieron interrumpidos por varios días, e incluso semanas. Inmediatamente después del terremoto, los saqueos fueron un grave problema en la región. De ellos fueron víctimas supermercados y grandes tiendas, al igual que como estaciones de servicio y pequeños comercios, al menos durante los primeros tres días, tras lo cual el Ejército se hizo cargo de la seguridad y hubo toque de queda por alrededor de un mes. La población se sintió en completo desamparo y las deficiencias en el actuar de las autoridades durante la emergencia, tanto a nivel regional como nacional, son fuente de debate hasta el día de hoy.

\section{Resiliencia comunitaria, capital social y lugar}

Durante los últimos quince años, la resiliencia comunitaria ha sido uno de los focos de estudio en la investigación sobre desastres, principalmente desde que se acordara el Marco de Acción de Hyogo 2005-2015, el principal acuerdo internacional para la reducción de riesgos de desastres, que promueve el aumento de la resiliencia de naciones y comunidades (Estrategia Internacional para la Reducción de los Desastres [EIRD], 2005). Para su definición y medición, últimamente se ha comenzado a considerar la relación entre distintos componentes de una comunidad, aunque aún no hay consenso respecto de si el concepto de resiliencia es solo aplicable a los sistemas sociales, o si es posible utilizarlo también en el caso de sistemas físicos o del medio construido. En esa disyuntiva, la mayor parte de la literatura existente sobre desastres considera de manera separada el capital social, por un lado, y el medio construido, por el otro.

Las definiciones de resiliencia están ligadas a los campos de estudio donde se emplea dicho concepto (González Muzzio, 2010). La primera definición fue propuesta por Holling en 1973, en un contexto ecológico, y luego han seguido muchos otros autores e instituciones (en el contexto de desastres, véase Mileti, 1999; Norris, Stevens, Pfefferbaum, Wyche \& Pfefferbaum, 2008; UNISDR, Resilience Alliance et al., en U.S. Indian Ocean Tsunami Warning System [IOTWS], 2006; entre otros). En general, las definiciones coinciden en señalar que una entidad es resiliente cuando tiene la capacidad de mantener y/o recuperar sus estructuras y funciones después de una perturbación. Algunos autores consideran que la resiliencia solo es aplicable a personas (Ganor \& Ben-Lavy, 2003), mientras otros apuntan a la interdependencia entre los sistemas sociales y los ecológicos (Folke et al., 2002 y 2005; por ejemplo). Los enfoques más integrativos sobre resiliencia, sin embargo, son recientes. Godschalk (2003) señala que una ciudad resiliente es "una red sustentable de sistemas físicos y comunidades humanas". Cutter, Boruff y Shirley (2003), por su parte, postulan que tanto el contexto geográfico como el tejido social determinan la vulnerabilidad de un lugar frente a un potencial desastre, entendiendo 'vulnerabilidad' como concepto opuesto a la resiliencia.

Una comunidad urbana es un sistema donde los ambientes construido, social, natural y económico interactúan y se influencian uno al otro (Norris et al., 2008, 
p. 128). Cutter et al. (2008b, p. 2) definen la resiliencia comunitaria en el contexto de la gestión de desastres como la "habilidad de un sistema humano de responder y recuperarse. Incluye aquellas condiciones inherentes al sistema que le permiten absorber impactos y enfrentar el evento, así como los procesos adaptativos posteriores que facilitan la capacidad del sistema de reorganizarse, cambiar y aprender en respuesta al evento", definición empleada en este caso.

El nivel de resiliencia de base o inherente de una ciudad (Rose, 2004) no es necesariamente un indicador de su grado de recuperación con posterioridad a un desastre, pues qué tan resiliente sea variará en función de la severidad del evento (Tierney, 2009) y de las respuestas iniciales de la comunidad (Cutter et al., 2008a). En este sentido, se debe considerar que planificar una ciudad cuyo soporte físico se presuma "resiliente", no necesariamente garantiza una rápida recuperación de la misma tras un desastre.

Cutter et al. (2008a) publicaron un modelo de resiliencia del lugar donde ocurre o puede ocurrir un desastre, conformado por el medioambiente construido, el sistema natural y el sistema social (Figura 1). Dicho modelo plantea que con anterioridad a la ocurrencia de un evento, el lugar presenta un nivel de vulnerabilidad y un grado de resiliencia inherentes al conjunto de sus componentes, que incluyen las dimensiones ecológica, social, económica, institucional, de infraestructura y de competencias de la comunidad. Cuando el evento sucede, presenta determinadas características y causa efectos inmediatos que gatillan respuestas de la comunidad. La capacidad que esta tiene para absorber el impacto puede o no verse sobrepasada por los efectos del desastre y las respuestas iniciales. Cuando no es sobrepasada, el grado de recuperación (y su rapidez) es alto. Pero si la capacidad de la comunidad de absorber impactos es sobrepasada, se requiere de resiliencia adaptativa, es decir, de respuestas dinámicas que los autores del modelo caracterizan como innovadoras y de permanente aprendizaje.

El modelo de Cutter y sus colegas constituye una de las escasas aproximaciones a la relación entre los sistemas construido, natural y social en la resiliencia comunitaria posdesastre. A nivel de barrio, en tanto, su papel en la resiliencia fue reconocido por Wallace y Wallace (2008), quienes enfatizaron su importancia debido a que corresponde al "nivel crítico de organización entre el nivel individual o familiar, la municipalidad y la región metropolitana”.

Las características o propiedades de una comunidad resiliente son un aspecto sobre el que no existe pleno acuerdo en la literatura. No obstante, una de las propuestas más citadas es la del MCEER (Multidisciplinary Center of Earthquake Engineering Research), que identifica cuatro propiedades aplicables a los sistemas físicos y a los sociales (Cuadro 1).

El capital social se entiende acá como un "activo multinivel que comprende la participación de individuos en grupos formales o informales o redes en la comunidad, así como las relaciones entre ellos y con las instituciones establecidas" (González-Muzzio, 2010). Dynes (2002) señala que este es el único tipo de capital que puede aumentar producto de un desastre. Norris et al. (2008) plantean que el capital social consiste en una serie de capacidades adaptativas que se refieren a las estructuras 
sociales y a la red de interconexión entre ellas; al soporte social, entendido como la ayuda percibida y/o recibida; y al sentido de comunidad y apego al lugar. Esto último puede influir tanto positiva como negativamente en la respuesta posdesastre.

FIGURA 1 | Modelo de 'Resiliencia del Lugar frente a los Desastres' (DROP Model)

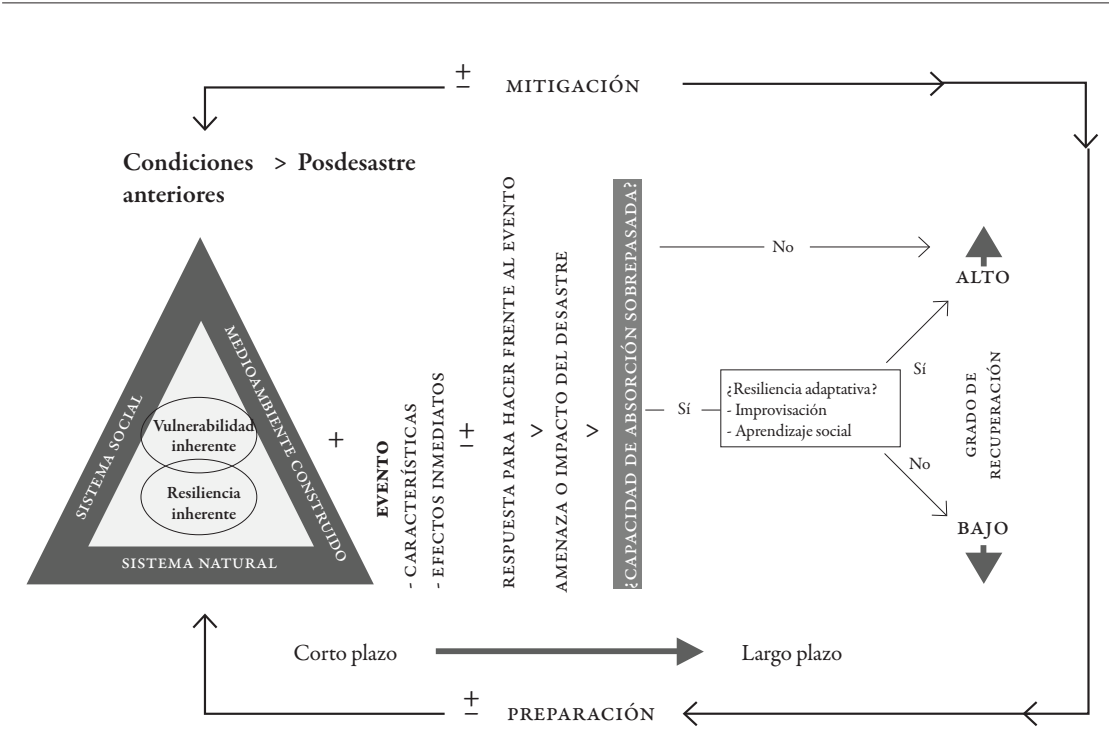

FUente CUtTer et AL., 2008A, P. 602. ORIGinal EN INGLÉs.

* DROP, Sigla en Inglés para DiSASTER RESILIENCE OF PLACE.

CUADRo 1 | Propiedades de una comunidad resiliente

\begin{tabular}{l|l}
\hline PROPIEDAD & DESCRIPCIÓN \\
\hline Robustez & $\begin{array}{l}\text { La habilidad de los elementos, sistemas y otras unidades de análisis de soportar } \\
\text { presiones o demandas sin sufrir daño, degradación o pérdida de función. }\end{array}$ \\
Redundancia & $\begin{array}{l}\text { La medida en que los elementos, sistemas y otras unidades de análisis pueden cumplir } \\
\text { funcionalmente con los requerimientos en caso de disrupción, degradación o pérdida } \\
\text { de función de los sistemas primarios. } \\
\text { La capacidad para identificar problemas, establecer prioridades y movilizar recursos } \\
\text { para evitar o enfrentar el daño o la disrupción; la habilidad de emplear recursos } \\
\text { materiales y humanos para satisfacer las prioridades y cumplir metas. }\end{array}$ \\
Rapidez & \begin{tabular}{l} 
La capacidad de satisfacer las prioridades y cumplir metas de una manera oportuna. \\
\hline
\end{tabular} \\
\hline
\end{tabular}

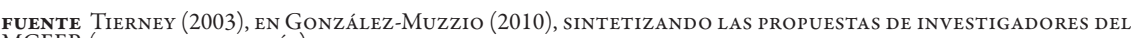
MCEER (ORIGINAL EN INGLÉS). 
En cuanto al capital social y la respuesta institucional posdesastre, investigadores del Centro de Investigación de Desastres de la Universidad de Delaware caracterizan la respuesta institucional y organizacional en función de su estructura y las actividades que llevan a cabo, diferenciando a instituciones y organizaciones según si dicha estructura y actividades se mantienen o cambian respecto de la situación previa al desastre. Quarantelli (2004) señala que, luego de un desastre, la creación de capital social y la aparición de grupos emergentes, así como el comportamiento emergente de instituciones y organizaciones existentes, son inevitables debido a la necesidad de acción inmediata después de ocurrido un evento. Es importante considerar la aparición de organizaciones y el comportamiento que surge o "emerge" durante el período posdesastre, porque ambos son reflejo de la flexibilidad y adaptabilidad de la comunidad a la nueva situación y de su capacidad para gestionar recursos acordes a los problemas que debe enfrentar.

Por su parte, el papel de las redes sociales y de las tecnologías de información y comunicación en la respuesta comunitaria posdesastre comenzó a ser investigado recientemente. Sutton, Palen y Shklovski (2008) consideran que el uso de los medios de comunicación y las redes sociales en el contexto de los desastres promoverán cambios más amplios en la respuesta comunitaria. Sin embargo, al momento de esta investigación no había estudios en áreas donde los sistemas y redes de telecomunicaciones hubieran fallado luego de ocurrido un evento. Aun así, tanto la Cruz Roja como Facebook reconocieron que las redes sociales y los medios de comunicación fueron importantes para mejorar la respuesta de la comunidad después de los terremotos de Chile y Haití (Cruz Roja, 2010; Inside Facebook, 2010).

\section{Área de estudio}

Para analizar la importancia del lugar y el capital social en la resiliencia comunitaria posdesastre, se tomó la ciudad de San Pedro de la Paz en el Área Metropolitana de Concepción (AMC) como caso de estudio.

El AMC tiene actualmente más de un millón de habitantes. En los últimos años, se ha caracterizado por un proceso de expansión urbana acelerado que ha implicado pasar de 4.747,121 hectáreas urbanizadas en 1974 (Smith \& Romero, 2009) a más de 10.000 hectáreas construidas en la actualidad. Producto de la continua expansión urbana, se han ido urbanizando áreas de alto valor ambiental, incrementando además la exposición de la población a peligros naturales debido a la ocupación desmedida de áreas inundables - principalmente humedales-, el borde costero y cerros.

San Pedro de la Paz se ubica en la ribera sur del río Biobío, frente a la ciudad de Concepción. La población comunal se estimaba al año 2009 en 93.241 habitantes (Subsecretaría de Desarrollo Regional y Administrativo [Subdere], 2010). Fue nombrada "comuna" en 1995, aunque el origen de la urbanización del área está vinculado al terremoto de 1960, luego del cual se construyó la Villa San Pedro con el fin reducir el déficit de vivienda ya existente en el área de Concepción y agravado por dicho terremoto (Franck \& Pérez, 2009). A partir de los años setenta, el proceso de crecimiento urbano en la comuna ha sido continuo, motivado primero por la existencia de terrenos disponibles a bajo costo y luego por la oferta de áreas no urbanizadas en un "entorno ambiental privilegiado". Aunque actualmente la comuna 
cuenta con supermercados, colegios y equipamiento básico repartido en distintos subcentros, sigue siendo predominantemente residencial y altamente dependiente del resto del AMC. Al 2002, un 56\% de los habitantes de San Pedro estudiaba o trabajaba en otras comunas del AMC (Instituto Nacional de Estadísticas [INE], 2002). Se trata de una comuna con un alto grado de segregación socioespacial, caracterizada por la presencia de condominios orientados a población de altos ingresos, localizados principalmente en las áreas altas, más centrales y mejor equipadas de la comuna, que contrastan con la existencia de poblaciones que surgieron inicialmente de la erradicación de población de bajos ingresos desde otros sectores del AMC, principalmente desde Concepción, y que se ubican en las áreas bajas y más alejadas de los centros de servicios.

El terremoto de 2010 no causó mayores daños en las edificaciones e infraestructura de la comuna, que tampoco fue afectada por el tsunami. No obstante, quedó semiaislada por varios días, debido a los daños en los puentes que cruzan el río Biobío y en las rutas a Santa Juana y Coronel (Figura 2), siendo objeto de saqueos masivos durante la primera semana que siguió al sismo. Se vieron afectados supermercados, estaciones de servicio, centros comerciales (por ejemplo, el Versluys), bodegas de alimentos, farmacias, restaurantes y pequeños comercios, tanto nuevos como antiguos, en distintos sectores de la comuna. Sanzana (2010) señala que San Pedro siguió a Concepción en cuanto a las denuncias de robo con fuerza, que alcanzaron a 102 en 2010, en comparación con 52 en 2009 en la misma comuna. No se indica, sin embargo, cuántos de ellos corresponden específicamente a saqueos.

Figura 2 San Pedro de la Paz en el contexto del AMC

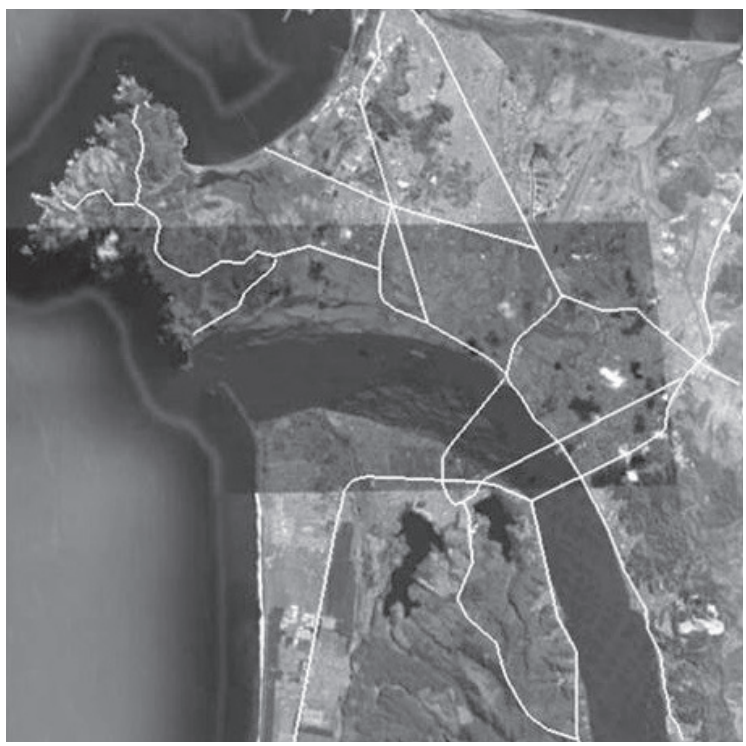

FUENTE ELABORACIÓN PROPIA SOBRE IMAGEN GOOGLE EARTH ${ }^{\circ}$. LAS LÍNEAS CORRESPONDEN A VIALIDAD PRINCIPAL INTERCOMUNAL. 


\section{Metodología}

Se busca comprender la importancia que tuvieron tanto el capital social existente y el generado tras el terremoto, como la configuración espacial del medio construido y los recursos presentes en el territorio, en cuanto a facilitar o dificultar la resiliencia de la comunidad urbana con posterioridad al desastre, durante el período correspondiente al primer mes luego de ocurrido el evento. Lo anterior, tomando en consideración las particulares circunstancias que siguieron al terremoto en San Pedro de la Paz, y que incluyeron el aislamiento físico, la caída de los sistemas de telecomunicaciones, falta de servicios básicos por un período prolongado y la ocurrencia de saqueos ${ }^{1}$ masivos.

Los datos se obtuvieron y analizaron considerando metodologías cuantitativas y cualitativas en conjunto. Se realizaron quince entrevistas en profundidad, una encuesta en línea y una revisión exhaustiva de prensa, así como del contenido de posteos en redes sociales, a través de Internet.

Las entrevistas se realizaron a dos distintos grupos de informantes: miembros de instituciones de nivel comunal a cargo de labores relacionadas con la emergencia, e informantes clave de la sociedad civil, tales como comerciantes, voluntarios y vecinos. Las entrevistas tuvieron una duración de entre cuarenta minutos y dos horas treinta minutos, y se llevaron a cabo durante una semana en junio de 2010. Se incluyeron preguntas cerradas y abiertas relativas al capital social existente y creado luego del terremoto, los problemas enfrentados por la población, los recursos básicos existentes en la comuna y su disponibilidad para ser utilizados durante la emergencia, y las actividades llevadas a cabo por cada entrevistado (o su institución, si correspondía) luego del terremoto. Respecto del perfil de los entrevistados, tenían entre 18 y 75 años, once hombres y cuatro mujeres, y varios de ellos ocupaban cargos en instituciones ligadas al manejo de la emergencia.

La encuesta en línea estuvo abierta entre el 19 de junio y el 18 de julio de 2010. Fue contestada por 112 personas, con 47 respuestas completas (42\% del total). Para invitar gente que respondiera la encuesta se hizo un posteo en Twitter, así como en grupos y páginas de Facebook relacionados con el terremoto en la comuna. De quienes completaron la encuesta, veintiséis tenían entre 26 y 40 años, catorce tenían entre 40 y 65 años y siete tenían 25 años o menos. De ellos, veintitrés son mujeres y veinticuatro, hombres.

Tanto las encuestas como las entrevistas consideraron tres cortes temporales en cuanto a problemas identificados, forma de enfrentarlos y otras preguntas relativas a la emergencia; esto es, las primeras cuarenta y ocho horas, la primera semana y el primer mes luego del terremoto.

1 En el análisis de situaciones de emergencia posdesastre se distingue entre "apropiación" y "saqueo". El primer concepto refiere a gente en situación de necesidad que toma comida y otros insumos básicos desde tiendas y supermercados, en especial cuando estos han sufrido daños. Tierney (2009) y Quarantelli (2007) reconocen que ambas situaciones ocurren luego de un desastre. Pero en este caso se habría tratado principalmente de saqueos, ya que sucedieron durante las primeras horas y días que siguieron al terremoto, cuando no existía "necesidad" urgente de apropiarse de alimentos u otros víveres, ni de obtener televisores, lavadoras u otros artículos que no son "de primera necesidad”. 
Por su parte, la revisión sistemática de prensa y posteos en redes sociales tuvo dos objetivos. El primero, obtener información del contexto en que se desarrollaron los hechos con posterioridad al terremoto en la comuna, considerando la escasez de información confiable o estudios sistemáticos desarrollados en el área a la fecha de la investigación (entre mayo y julio de 2010). El segundo, medir la importancia que tuvieron las redes sociales en la respuesta de la comunidad y explorar su influencia en la formación o fortalecimiento del capital social local. Se revisó un diario de circulación nacional (La Tercera) y uno regional (El Sur) en sus versiones online, entre el 27 de febrero y el 31 de marzo, así como el contenido de páginas y grupos en Facebook y tweets relacionados con San Pedro de la Paz y el terremoto durante el mismo período.

Si bien el bajo porcentaje de respuestas completas en la encuesta dificulta un análisis estadístico preciso, los datos proporcionados por quienes la contestaron fueron plenamente coincidentes con lo planteado por los entrevistados (a quienes se hicieron las mismas preguntas cerradas). Los entrevistados detallaron las situaciones vividas y la forma de enfrentar los problemas de los que debieron hacerse cargo desde sus distintas responsabilidades, permitiendo de este modo explicar y contextualizar las respuestas, al igual que se hizo con la revisión de las redes sociales y periódicos. La síntesis de resultados que se presenta a continuación, por tanto, contempla información obtenida a través de los distintos métodos utilizados.

\section{Resultados}

Los problemas que debió enfrentar la población de San Pedro de la Paz durante el primer mes luego del terremoto variaron con el correr del tiempo (Gráfico 1). Durante las primeras cuarenta y ocho horas, los principales problemas fueron la falta de acceso a telefonía móvil, la falta de agua potable y electricidad, y la delincuencia o saqueos. La falta de agua, electricidad, alimentos y combustible constituyeron las principales dificultades durante la primera semana, mientras que los saqueos fueron disminuyendo en importancia en dicho período luego que los militares quedaran a cargo de la seguridad en la región. A los problemas que persistieron se sumó, entonces, la falta de conectividad con Concepción. Al final del primer mes esta situación no había sido solucionada, y a ella se sumaba el que ni colegios ni escuelas, y tampoco el transporte público, habían podido reanudar sus actividades. La falta de conectividad con Concepción siguió siendo un problema al momento de la investigación, y a él los entrevistados añadían la pérdida de empleos.

De los problemas enfrentados, la carencia de agua potable, alimentos y combustible, la falta de electricidad y el temor a la delincuencia y saqueos, fueron enfrentados principalmente de forma colaborativa durante las primeras cuarenta y ocho horas y al menos hasta la primera semana luego del terremoto. Al final del primer mes, las actividades realizadas en forma colaborativa disminuyeron en cantidad e importancia. De manera creciente se mencionó entre los problemas más relevantes enfrentados colaborativamente en el último periodo, la falta de conectividad 
con Concepción (paliada compartiendo vehículo) y el cuidado de los niños. Un entrevistado aclaró que, al final del primer mes, "aún muchos colegios no habían comenzado el año escolar y la mayoría de los papás ya estaban trabajando, así que nos organizábamos entre los mismos vecinos".

Los factores que gatillaron la organización comunitaria fueron consultados a los entrevistados, quienes señalaron como principales razones el temor a los saqueos, la carencia de servicios básicos y comida y la falta de apoyo por parte de las autoridades.

GRÁFICO 1 | Principales problemas enfrentados por la comunidad durante las primeras cuarenta y ocho horas, primera semana y hasta el final del primer mes luego del terremoto

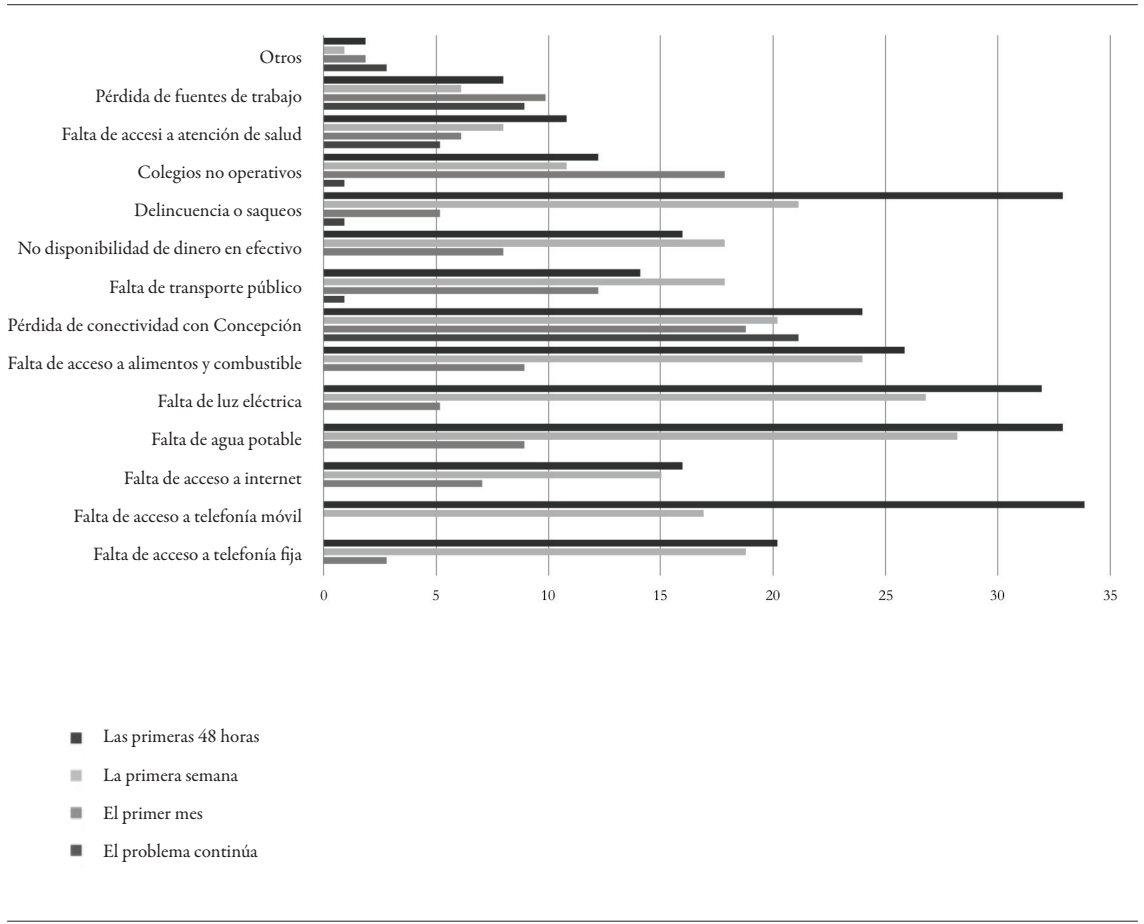

FUENTE MODIFICADO DE GoNZÁleZ-MuZZio (2010).

\section{Capital social pre y posdesastre}

Casi la mitad de los encuestados participaba en organizaciones formales con anterioridad al desastre, principalmente en una sola organización. Algunos mencionaron que la participación previa en organizaciones sociales era mayor en la población más pobre, "acostumbrada a organizarse por el tema de los subsidios" (funcionaria de municipio, com. pers., junio 2010). No obstante, se produjo un aumento en la participación de población de otros estratos socioeconómicos en organizaciones 
formales después del terremoto, situación que hicieron notar encuestados y entrevistados. Algunos comenzaron a participar en grupos formales, como Un Techo para Chile, además de su integración a las organizaciones de voluntariado informal que se establecieron en la comuna los días posteriores al 27 de febrero.

Si bien la gente se organizó colaborativamente luego del desastre, el alcance de la organización varió fuertemente durante el primer mes, tanto en términos de sus miembros como de su extensión territorial (Gráfico 2). La familia y los vecinos de la misma cuadra o pasaje fueron las principales formas de organización durante las primeras cuarenta y ocho horas. Durante la primera semana, en tanto, los vecinos de la misma cuadra fueron los más mencionados, seguidos de los amigos y después la familia. Por su parte, los grupos en los que los consultados participaban con anterioridad al evento fueron aumentando en importancia con el correr de los días; sin embargo, estos fueron siempre menos relevantes que la familia y que los grupos de vecinos que emergieron luego del terremoto.

GRÁFICO $2 \mid$ ¿Entre quiénes se compartieron responsabilidades para enfrentar las consecuencias del desastre?

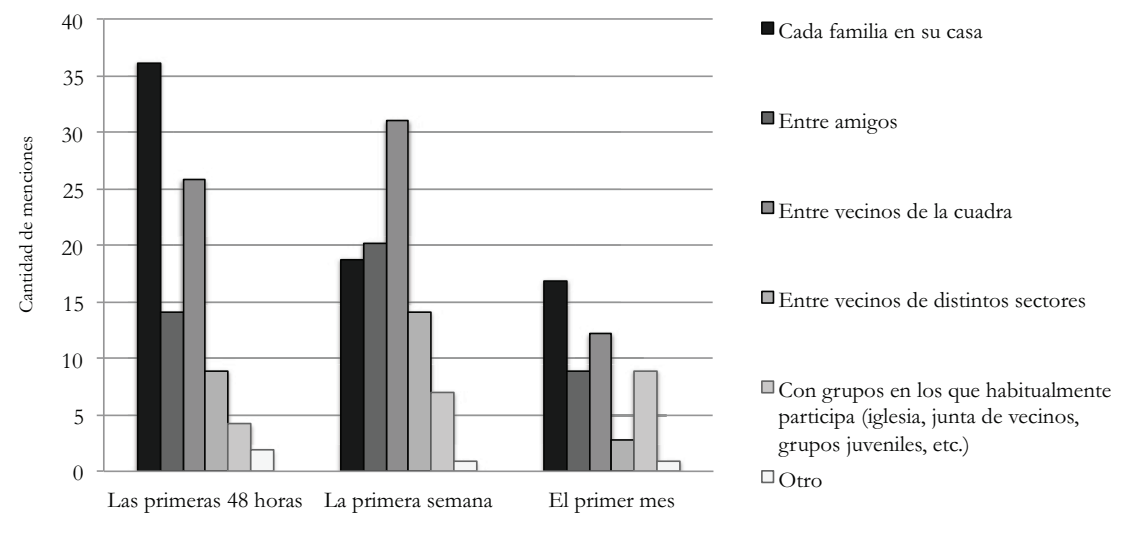

FUENTE MODIFICADO DE GONZÁlEZ-MuZZio (2010).

Gran parte de los encuestados (33 de 47) y entrevistados (13 de 15) participaron en grupos de vecinos organizados tras el terremoto. Quienes no se involucraron señalaron vivir en calles donde ello no fue necesario, porque eran constantemente transitadas por militares, considerándose áreas seguras, o bien porque "la calle era muy ancha para poder cerrarla", o porque no viven en la comuna. Algunos entrevistados señalaron que se incluyó en los grupos de pasajes a quienes enfrentaban las avenidas, para que no quedaran aislados y recibieran ayuda del municipio, ya que la distribución de ayuda se hizo principalmente en función de la organización vecinal generada, y no puerta a puerta ni a través de las organizaciones formales preexistentes, como juntas de vecinos. 
Aunque en su mayor parte los grupos emergentes estuvieron activos durante la primera semana o el primer mes después del $27 / \mathrm{F}$, en algunos casos se conserva una relación de amistad entre vecinos que se ha profundizado con el tiempo. Muchos señalaron que fue "lo bueno del terremoto".

Otros grupos que emergieron después del terremoto en San Pedro de la Paz o relacionados con la localidad fueron voluntarios, grupos en redes sociales y "saqueadores".

Respecto del voluntariado, inmediatamente después del evento muchos se acercaron al municipio ofreciendo colaboración. En un principio fue la gente de Villa San Pedro y Huertos Familiares, sectores más cercanos al municipio, sumándose luego personas de otras áreas de la comuna, hasta alcanzar alrededor de trescientos voluntarios que participaron regularmente. El municipio fue el primer centro de operaciones, pero luego la cantidad de ayuda y voluntarios aumentó, por lo que una semana después el grupo se trasladó al gimnasio municipal, donde se acopió la ayuda. Durante la primera semana, los voluntarios realizaron actividades en la comuna, mientras que al final del primer mes las labores de voluntariado continuaban, pero principalmente fuera de ella. Lo anterior se explica porque los requerimientos de largo plazo en San Pedro eran mínimos (en términos de viviendas dañadas o destruidas y cantidad de empleos perdidos) en comparación a lo que sucedía al norte del Biobío, en áreas como Talcahuano y Chiguayante, entre otras. Otro factor que influyó fue la conectividad hacia Concepción, que fue mejorando con el correr del tiempo.

Tanto Twitter como Facebook fueron clave en facilitar la respuesta de la comunidad en San Pedro de la Paz y otros lugares afectados, a pesar del apagón de telecomunicaciones ocurrido tras el evento y la falta de energía eléctrica, que se mantuvo al menos durante la primera semana en la mayor parte de la comuna. Dentro y fuera de San Pedro hubo quienes lograron acceder a redes sociales y coordinar apoyo externo, así como proveer información a los vecinos. Un entrevistado expone: "Mucha gente vive en San Pedro, pero no es tan grande. Uno puede hablar de varios de los lugares porque los conoces o tienes amigos ahí.... Para lograr mayor eficiencia en la distribución de la información, otro explica que "si alguien sabía o quería información sobre un sector, pasábamos el comentario a alguien de ese barrio... para que informara directamente". Varios puntualizaron que cargaban los celulares con los generadores de los bomberos, corriéndose también la voz a través de las redes sociales. Luego del terremoto, casi la totalidad de los encuestados utilizó una o más redes sociales, dieciséis de ellos solo durante los primeros días. Hubo una activa participación en grupos de Facebook relacionados con San Pedro de la Paz durante el primer mes posdesastre, contándose entre los principales los denominados "Yo soy voluntario en San Pedro de la Paz" (344 miembros) y "Qué está pasando en San Pedro de la Paz" (369 miembros), relacionados directamente con la emergencia.

Otro tipo de grupos emergentes surgidos después del terremoto tiene relación con los saqueos sucedidos a continuación del evento. Estos grupos fueron mencionados por encuestados y entrevistados, incluyendo carabineros y comerciantes afectados. Uno de ellos señaló: "Vinieron en micros, con pistolas, media hora después del terremoto. ¿Qué íbamos a hacer? Los dejamos llevarse todo”. Otro indicó 
que en su pasaje muchos se organizaron apenas terminó el sismo, aprovechando la oscuridad y el caos, para saquear locales cercanos. En su mayoría se trataría de vecinos de poblaciones de bajos ingresos; no obstante, hubo sectores donde se sumaron espontáneamente habitantes de distintos estratos socioeconómicos, incluidos vecinos de barrios de altos ingresos. Bullado fue el caso de un ingeniero sorprendido llevando mercaderías en su vehículo, desde un supermercado. Asimismo, varios comerciantes afectados en diversos sectores de la comuna reconocieron a clientes y vecinos entre quienes allanaron sus propiedades. Vecinos de Boca Sur, uno de los sectores desde donde varios entrevistados afirman que provenían algunos de estos grupos "organizados", emitieron un comunicado público donde justifican el ingreso a supermercados debido a la incertidumbre y a la inacción del gobierno, señalando que se habían organizado para satisfacer sus necesidades.

En relación a los "saqueadores", aclaramos que no es responsabilidad de los vecinos/as esta situación. La inoperancia del Gobierno no la podemos pagar los más necesitados; la desesperación ante la incertidumbre de lo que pasará y la falta de alimentos obligó a muchas familias a ingresar a los supermercados y obtener alimentos para los suyos...

A pesar de la falta de respeto de la alcaldesa de Concepción y San Pedro de la Paz en calificar de "saqueadores" a los pobladores y pobladoras de Boca Sur y del borde costero de la comuna, la respuesta es la organización, solidaridad y unidad de los vecinos/as, los que se han organizado en guardias vecinales para defender los pasajes y calles, se han instalado comedores populares y se ha centralizado la información en una casa de la población que muestra la capacidad que tenemos los pobladores/as para cuidarnos y dar respuesta a nuestras demandas (www. radioplaceres.cl, 13 de marzo de 2010).

Cabe recalcar que, antes de los saqueos, en la comuna existía stock de mercaderías de diverso tipo, las que por causa de los latrocinios no estuvieron disponibles para la gran mayoría de los habitantes durante los primeros días después del sismo, provocando el desabastecimiento percibido por la comunidad.

\section{La estructura física de las urbanizaciones fue determinante en la organización social}

Un aspecto relevado por encuestados y entrevistados es que la organización comunitaria se basó en la proximidad espacial de los vecinos. Uno señaló que la organización era principalmente por pasajes o calles pequeñas.

Tuvo que ver con las calles chicas y no con las grandes. San Pedro está compuesto por muchas villas... Bueno, por ejemplo, en el sector de Los Pioneros, también está lleno de pasajes y condominios y los vecinos cerraron ahí. En el sector de Los Huertos cerraron estructuras grandes, porque las calles son avenidas y cerraban aquí y... vigilaban.

Otro entrevistado puntualizó que existieron distintas escalas en la organización: las cuadras o condominios, varias manzanas o condominios cercanos, y una 
mayor que podía involucrar incluso a la junta de vecinos. La organización comunitaria consideró principalmente la proximidad entre las viviendas y no la existencia previa de lazos entre vecinos.

De este modo, la forma urbana de las poblaciones, barrios y condominios habría contribuido a la masiva organización vecinal. Sin embargo, varios entrevistados señalaron que la estrechez de calles y pasajes, así como lo intrincado del tejido urbano en muchas poblaciones o villas y la carencia de espacios públicos amplios, dificultó el proceso de evacuación, aumentando la vulnerabilidad de la población ante amenazas de origen antrópico (como incendios) o ante la posibilidad efectiva de un maremoto, en especial en sectores de bajos ingresos.

\section{Influencia de las características del área en los efectos del terremoto y tsunami y en la respuesta de la comunidad}

Los daños provocados directamente por el terremoto en San Pedro de la Paz fueron sustancialmente menores en comparación con otras comunas del AMC: el tsunami no afectó las costas de la comuna ni las riberas del río Biobío, probablemente por la posición de la costa de San Pedro en el golfo de Arauco y en relación con la península de Hualpén. En su mayor parte, los daños en edificaciones e infraestructura ocurrieron en áreas de relleno sobre sectores del humedal Los Batros, en rellenos en el camino a Santa Juana o en terrenos arenosos, situación que puso en el tapete la inconveniencia de urbanizar sobre humedales y otros sectores ambientalmente sensibles, no solo por la pérdida de patrimonio ambiental y el impacto sobre dichos ecosistemas, sino también por su condición inherente de vulnerabilidad frente a terremotos e inundaciones.

Al ser consultados por las áreas más vulnerables y las más seguras frente a terremotos y tsunamis, la mayoría coincidió en señalar los sectores de Boca Sur, Michaihue y San Pedro Costa como áreas vulnerables, por ser zonas costeras o ribereñas situadas en elevaciones bajas. En cuanto a las áreas seguras, gran parte de la población recurrió a los lugares que, desde el denominado "falso tsunami" en enero de 2005 , quedaron en la memoria colectiva. Estas corresponden a los sectores más altos de la comuna que presentan accesibilidad vehicular y espacios públicos amplios, principalmente Andalué e Idahue, ambos habitados mayoritariamente por población de estratos socioeconómicos medio-alto y alto. Lo anterior, aun cuando no fueran esas las áreas más cercanas a todos los domicilios. Así, otras áreas elevadas, como un sitio eriazo conocido como cerro Chico, o el cerro del Anfiteatro, no fueron utilizadas durante la evacuación. Gran parte de la población comunal confluyó entonces a Andalué e Idahue, con la excepción de los vecinos del sector Lomas Coloradas, más alejado del centro de la comuna, desde donde la población evacuó hacia los cerros al oriente de la ruta 160 a Coronel.

La falta de conocimiento de los vecinos respecto de las áreas potencialmente afectadas por inundaciones causadas por un maremoto, más allá de las zonas costeras propiamente tales, provocó que muchos evacuaran sus casas sin que fuera necesario hacerlo, aumentando la congestión en calles y áreas seguras. Incluso a la fecha de la investigación, 
eran pocos los vecinos que conocían cuáles eran las áreas hacia las cuales debían evacuar en caso necesario, a pesar de existir un mapa generado por bomberos y otros organismos con posterioridad al sismo, el cual fue difundido por una revista comunal.

Respecto del agua potable, la escasez fue evidente durante los primeros días, agravada por los saqueos y luego por la tardía restitución del servicio. La mayoría de los sampedrinos, sin embargo, accedió a agua utilizando los recursos existentes en el lugar. Las lagunas, fácilmente al alcance de la comunidad, fueron las primeras fuentes utilizadas tanto para el baño como para el lavado, e incluso para cocinar, pero también se obtuvo de vertientes situadas en laderas de cerros y punteras. Este último sistema, que extrae agua directamente desde las napas subterráneas, fue de uso masivo en la comuna, ya que en muchos terrenos las napas se encuentran a poca profundidad y existían muchas punteras en funcionamiento con anterioridad al terremoto.

De la misma forma, aquellos que contaban no solo con conocimientos previos del lugar en sí, sino con una identificación consciente de las áreas seguras, evacuaron hacia sectores más cercanos a sus viviendas y menos concurridos por la población en general. Y de igual modo, algunos decidieron no evacuar por considerar que frente a una inundación por tsunami no se verían afectados.

Los sampedrinos están conscientes de la fragilidad que presenta la comuna en cuanto a su conectividad tanto al norte como hacia el sur del Biobío. La disminuida accesibilidad hacia Concepción con posterioridad al desastre afectó tanto las posibilidades de aprovisionamiento de la comuna como el acceso al trabajo desde y hacia la misma. El servicio de salud municipal, por ejemplo, trabajó los primeros días con alrededor de quince o veinte personas de un total aproximado de cuatrocientos funcionarios, porque muchos de ellos viven en otras comunas del AMC (director del Centro de Salud Familiar en Candelaria, com. pers., junio 2010). Otro efecto observado es que, con posterioridad al desastre, varios vecinos cambiaron a sus hijos a colegios situados en la comuna, debido a los problemas de transporte hacia Concepción y por el temor a quedar nuevamente aislados y las familias separadas.

La oferta de servicios educacionales, comercio y otros servicios de escala local, cada vez más abundantes y diversos durante los últimos quince años, se ha concentrado principalmente en el centro histórico de la comuna, pero también en las cercanías de las nuevas urbanizaciones (Figura 3). En el contexto metropolitano, sin embargo, San Pedro de la Paz sigue siendo considerada como una comuna dormitorio (Pérez \& Salinas, 2007). Los sampedrinos reconocen la diversidad de usos como un activo a la hora de enfrentar un desastre; no obstante, en su mayoría estos recursos no estuvieron disponibles para la población durante los primeros días después del evento, especialmente debido a los saqueos. Por ello, fue clave la presencia de empresas y bodegas situadas en la comuna, desde donde provino gran parte de los alimentos que se repartieron a los vecinos durante las primeras semanas y que luego se entregaron también a otras comunas del AMC. Las dificultades para distribuir productos fuera de la comuna, la falta de la energía eléctrica necesaria para mantener refrigerados los alimentos y el temor a los saqueos, fueron factores que gatillaron la entrega de mercadería al municipio para su distribución. 
Figura 3 | Esquema de usos de suelo en San Pedro de la Paz

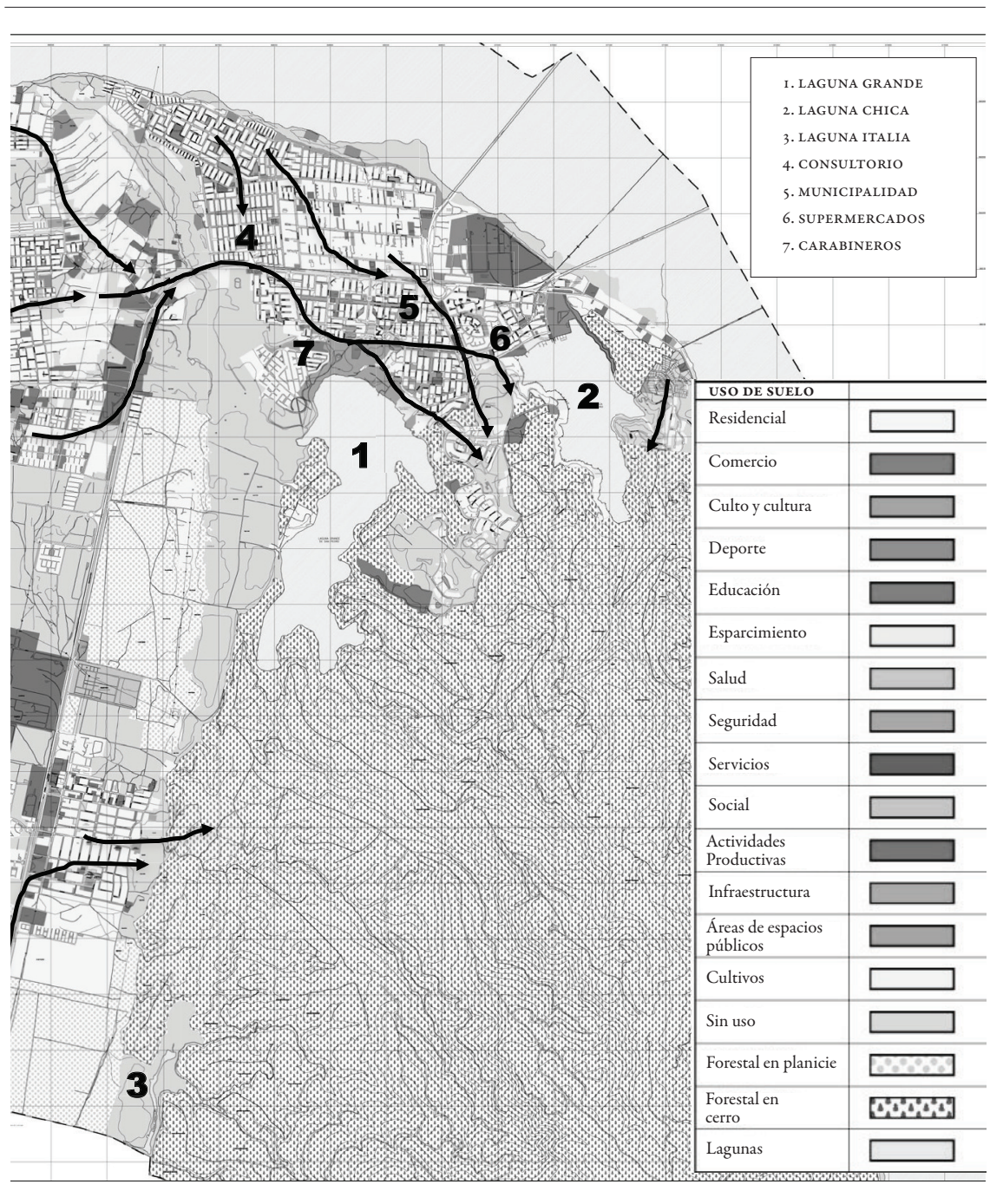

FUENTE GONZÁLEZ-MUZZio (2010), MODIFICADo DE DURÁN (2006). LAS LínEAS ESQUEMATIZAN LAS PRINCIPALES RUTAS DE EVACUACIÓN UTILIZADAS DESPUÉS DEL TERREMOTO.

\section{Discusión}

\section{Resiliencia inherente y resiliencia adaptativa}

El capital social (entendido como la participación de individuos en grupos o redes en la comunidad, así como las relaciones entre ellos y con las instituciones establecidas) y el lugar (correspondiente al medio natural y construido donde interactúa la comunidad) contribuyeron a la resiliencia de la comunidad urbana de San Pedro de la Paz, influenciándose mutuamente, tanto positiva como negativamente. 
Revisando lo ocurrido en San Pedro luego del terremoto, y aplicando para ello el modelo de Cutter et al. (2008a), es posible señalar que las condiciones existentes con anterioridad al desastre permitían prever una rápida recuperación de la comuna luego del desastre, debido a su alto nivel de resiliencia de base en relación con su medioambiente construido y los sistemas naturales, aunque respecto de los sistemas sociales la situación era disímil entre grupos de distinto nivel socioeconómico (Figura 4).

En el lugar existen áreas seguras a las cuales la población pudo acceder, a pesar de la inexistencia de un plan de evacuación en el área. Los potenciales efectos del tsunami en la comuna fueron mitigados por la península de Hualpén, evitando posibles daños por esta causa. Las edificaciones resistieron de forma adecuada y no hubo daños de consideración en infraestructuras críticas locales, como el municipio, los servicios de salud, colegios, etc. Además, existía suficiente stock de combustible y alimentos para soportar varios días en condiciones de aislamiento, en caso de que este se produjera.

Con anterioridad al terremoto, el capital social era dispar al interior de los distintos sectores en la comuna, especialmente en lo referido a organizaciones formales. Estas eran mucho más activas y abundantes en áreas de escasos recursos. Comfort (1999) reconoce que esta situación es común y señala que por tal razón los grupos de estratos bajos presentan una mayor resiliencia de base, a lo cual se suma la existencia de redes de apoyo más consolidadas. No obstante, pese a ser más resilientes socialmente, los grupos de estratos más bajos son considerados más vulnerables, debido a que físicamente se encuentran localizados en áreas más expuestas a riesgos y "porque sus reservas son más limitadas" (Godschalk, 2003). Sin embargo, lo observado en cuanto a los lazos informales en San Pedro de la Paz es que son heterogéneos, en especial entre la población joven que estudia y vive en la comuna, a lo cual han contribuido, además, las redes sociales.

Tras el sismo, el alto grado de resistencia de las edificaciones contrastó con lo ocurrido respecto de la infraestructura vial de nivel intercomunal, incluidos el colapso del Puente Viejo y los daños en los dos puentes que se encontraban en uso, que dificultaron o impidieron su utilización; hubo también derrumbes y cortes en el camino a Santa Juana y daños en el puente Los Batros camino a Coronel, todo lo cual dejó a San Pedro de la Paz semiaislado. Esta situación puso en evidencia cuán dependiente es la comuna de los bienes y servicios localizados en Concepción o en áreas más alejadas. También evidenció la necesidad de contar con equipamientos indispensables en áreas urbanas cercanas a los 100.000 habitantes, tales como hospitales. La infraestructura sanitaria, eléctrica y de telecomunicaciones, por su parte, presentó las mismas dificultades que en el resto de la región, tardando semanas en restablecerse con normalidad.

La resiliencia de base a nivel comunal fue modificada por las respuestas iniciales a los efectos del terremoto y por la situación de aislamiento, no siendo indicativa del nivel de resiliencia de la comunidad luego del desastre, lo que coincide con lo expuesto por Tierney (2009). A una respuesta positiva, como la autoevacuación, siguió una negativa, los saqueos. Como resultado, la capacidad de la comunidad para absorber impactos fue sobrepasada y se requirió de respuestas adaptativas de su parte y de las autoridades e instituciones de nivel local. 


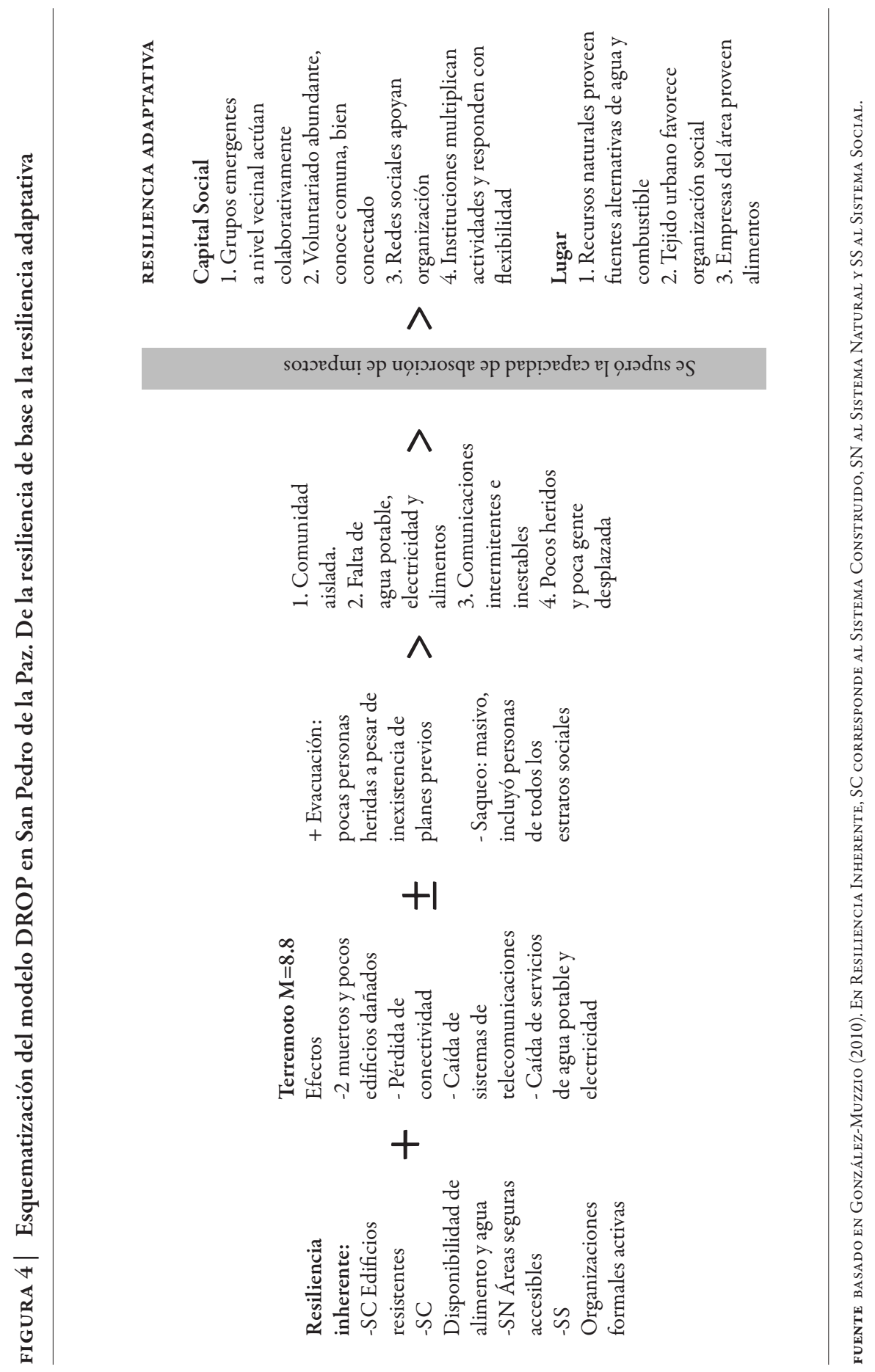


La creación de capital social nuevo en las distintas escalas, así como la modificación de estructuras institucionales y su comportamiento adaptativo o emergente, fueron las principales respuestas, siendo todas ellas consideradas respuestas "resilientes" (Tierney, 2009). En todos los niveles la población empleó los medios de comunicación disponibles, como radio, teléfonos y las redes sociales Twitter y Facebook, y las autoridades locales reconocieron el papel de los grupos sociales que emergieron tras el terremoto, lo que facilitó la distribución de ayuda en terreno. El papel de redes sociales y voluntarios fue clave, ya que se convirtieron en nodos que comunicaban información entre vecinos y entre estos y el comité de emergencia comunal.

Aunque no todos los grupos identificados presentaron conexiones entre sí, se observó que algunas personas participaron en más de un tipo de grupo, lo cual contribuyó a diseminar información entre grupos o individuos que se encontraban menos conectados y con menor acceso a la información (Figura 5). Sin embargo, esto solo fue considerado parcialmente por los encargados de la emergencia. Si bien se tomó en cuenta la formación de grupos a escala vecinal y se validó su existencia distribuyendo la ayuda a través de ellos, solo algunos funcionarios en cargos estratégicos accedieron a redes sociales y/o internet durante el período de la emergencia, por lo que desconocían las demandas y problemas que manifestaba la comunidad a través de esos medios. Se requiere conocer la interacción entre diversos grupos que actúan respondiendo a los requerimientos que presentan las emergencias, ya que las comunidades son dinámicas en cuanto a sus miembros y límites y muchas personas forman parte de más de una comunidad a la vez (Twigg, 2007).

Las capacidades adaptativas de la gente y su conocimiento del lugar facilitaron también su respuesta para proveerse de recursos alternativos o "redundantes" que se encontraban disponibles en el área, tales como agua y combustible (uso de leña), alimentos y medicinas, entre otros. Fue importante el hecho de que los sampedrinos eran conscientes de su potencial aislamiento con anterioridad al evento y, por lo tanto, no esperaban una rápida llegada de apoyo externo.

Por su parte, el tejido urbano influenció la respuesta comunitaria, por cuanto los grupos emergentes de vecinos coincidían en gran medida con cuadras, pasajes u otras tipologías de urbanización, como los condominios (Figura 6). Ello se materializó independientemente de la existencia de lazos previos entre los vecinos y permitió a los vecinos "rapidez" en la organización. El actuar colaborativamente facilitó un acceso más expedito a los recursos disponibles y su adecuada distribución y gestión. El tamaño de cada grupo y la decisión de organizarse o no fue influenciada por el largo y ancho de calles y pasajes, así como por la densidad habitacional de cada área.

Conceptos como "visibilidad" y "conectividad" fueron nombrados por algunos entrevistados como factores que podrían hacer que un área fuese más o menos segura frente a la llegada de "turbas de saqueadores", y a partir de ello decidían si cerrar o no el paso en un pasaje o calle determinada. El hecho de que en calles más anchas o transitadas la gente no se organizara es consistente con algunos estudios realizados por medio de análisis de sintaxis espacial, que señalan que el crimen es menos frecuente en áreas más integradas donde los flujos vehiculares y peatonales son mayores (Shu, 1999, en Greene \& Mora, 2008, p. 151), lo que hace dichas calles más seguras que los pasajes o vías con una menor continuidad e integración. 
FIGURA 5 | Principales grupos e instituciones involucrados en la gestión de la emergencia en San Pedro de la Paz

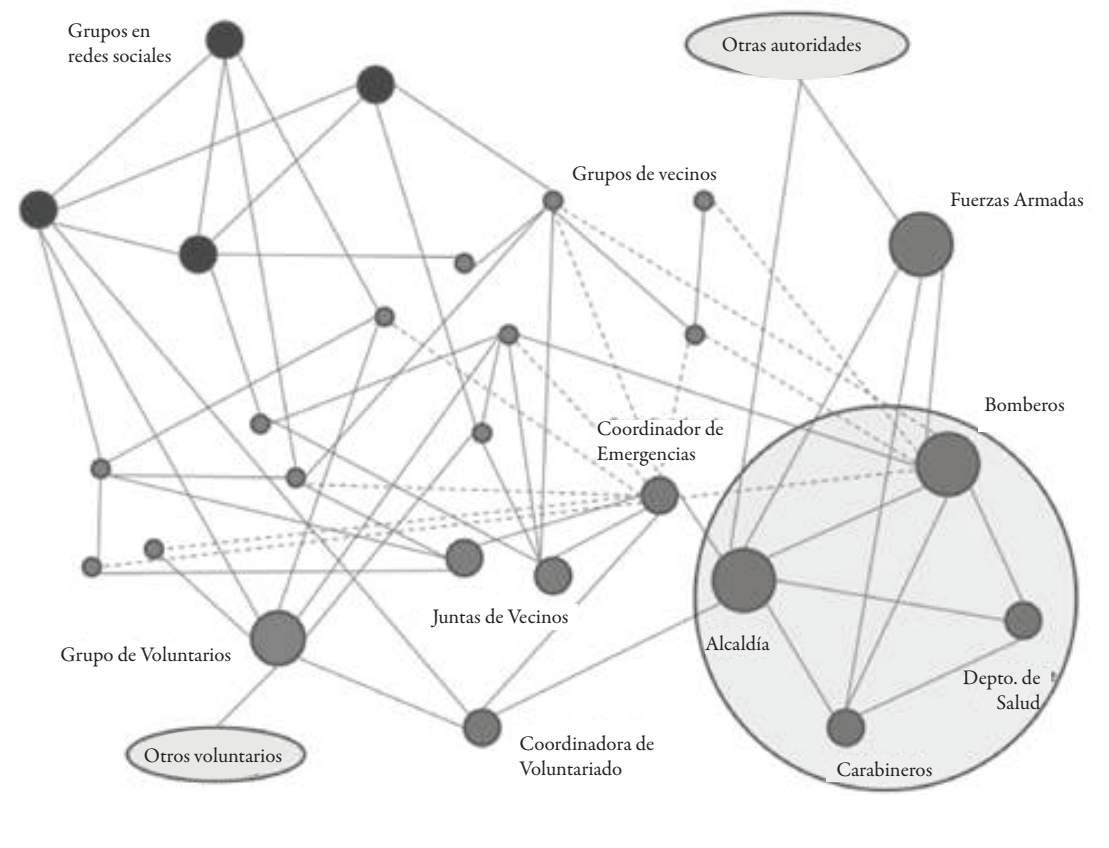

FUENTE GonZÁleZ-MuZzio (2010, P. 63). ORIGINAL EN INGLÉS.

FIGURA 6 | Ejemplificación de organización comunitaria en pasajes, condominios y grupos de condominios

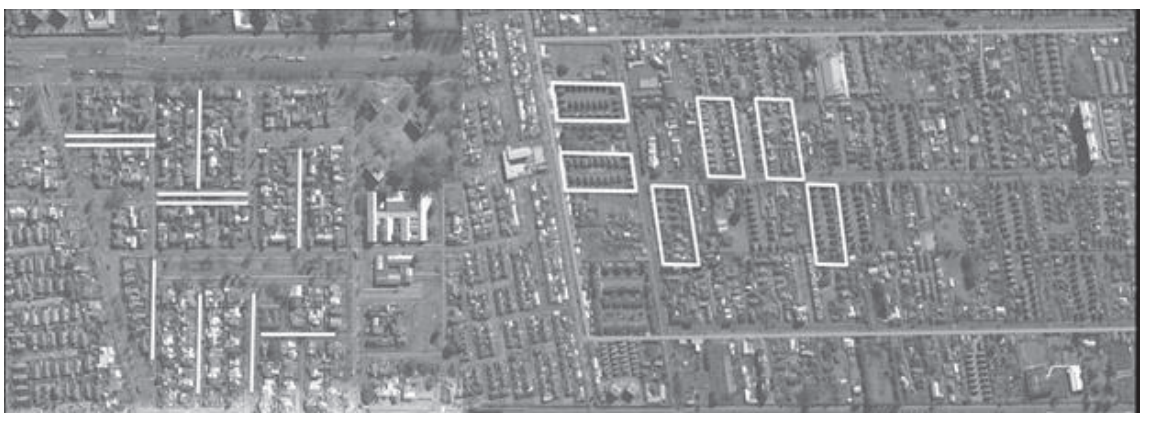

FUENTE MOdificado de GoNZÁleZ-MuZzio (2010)

El impacto de los desastres no se manifiesta solo sobre las edificaciones y la infraestructura. En San Pedro de la Paz, gran parte de las edificaciones resistieron el terremoto sin problemas, es decir, fueron "robustas". Se exceptuaron algunas cons- 
trucciones situadas en áreas vulnerables; por ejemplo, rellenos sobre humedales. Ello vuelve a poner en evidencia que el crecimiento urbano presenta limitaciones impuestas por las condiciones del territorio, y que estas deben ser consideradas en los procesos de planificación. Se debe restringir dichas áreas al desarrollo urbano o establecer condiciones para su ocupación que mitiguen los potenciales efectos de un evento extremo, disminuyendo la vulnerabilidad del lugar y de la comunidad.

Pero la planificación y reconstrucción de ciudades resilientes debe contemplar no solo la construcción de infraestructura física y edificaciones resistentes o robustas. Se tiene que identificar y proteger recursos que son clave durante emergencias que afecten el área urbana, y asegurar la posibilidad de acceder y hacer uso de recursos físicos y naturales "redundantes", que posibiliten respuestas rápidas y eficientes por parte de la comunidad. Esto es válido tanto en circunstancias de aislamiento, como en general durante los primeros días luego de ocurrida una emergencia, pues se reconoce a nivel mundial que son los propios afectados y los gobiernos locales los primeros en responder ante cualquier evento extremo. La ciudad debe ser capaz de proveer los recursos necesarios para la supervivencia de la comunidad, facilitando su rápida recuperación. La mixtura de usos existente en San Pedro de la Paz fue clave en este sentido, lo mismo que la existencia de espacios abiertos posibles de ser ocupados como áreas de evacuación.

Luego de ocurrido un desastre, es frecuente la aparición de grupos sociales cuyo objetivo es generar estas respuestas. En el caso de San Pedro de la Paz, muchos de estos grupos surgieron en función de las características del tejido urbano, incluidos los "grupos de saqueadores", situación que debe ser estudiada con mayor profundidad.

La creación de capital social negativo, manifestada en la aparición de grupos más o menos organizados que aprovecharon la emergencia para realizar los saqueos, tuvo como consecuencia que las capacidades de la comunidad para recuperarse rápidamente después del sismo fueran superadas, a pesar del alto grado de resiliencia inherente que el lugar tenía. No obstante, los grupos emergentes conformados por vecinos, voluntarios y los surgidos en las redes sociales, tuvieron conductas altamente adaptativas que les permitieron hacer frente a la nueva realidad, lo que fue facilitado por la existencia de una estructura espacial de villas, pasajes y condominios que posibilitaron la organización en grupos pequeños, pero también por la presencia de recursos redundantes. No obstante, también se puso en evidencia la necesidad de contar con otros servicios que permitieran una oportuna atención a los vecinos durante la emergencia, tales como recintos de salud de alta complejidad.

Por lo anterior, el proceso de reconstrucción y la implementación de estrategias de reducción de riesgo de desastres en las ciudades debe incluir la participación de los grupos sociales creados espontáneamente durante el período de emergencia, pues ellos pueden convertirse en el capital social de base frente a la ocurrencia de futuros eventos, contribuyendo a aumentar la resiliencia inherente del sistema urbano. Disminuir la segregación socioespacial y las inequidades respecto de la vulnerabilidad frente a las amenazas naturales, debe ir de la mano con la construcción de vecindarios que fomenten la generación de lazos entre vecinos y favorezcan la 
seguridad de los mismos. Al mismo tiempo, se debe procurar que los vecinos conozcan no solo los peligros a los que están expuestos, sino también los recursos disponibles en la comuna - o en el barrio - que les servirán para enfrentar posibles dificultades. Estas medidas permiten mejorar la resiliencia inherente de la ciudad; $\mathrm{y}$, al mismo tiempo, reconocen que dependiendo de la gravedad de los efectos de un evento, la población deberá responder en forma adaptativa y para ello hará uso de los recursos disponibles en el territorio.

Esta investigación no pretende ser conclusiva respecto de los temas expuestos, pues es altamente dependiente del contexto físico y temporal en el cual fue desarrollada. Se quiere, en cambio, abrir el debate respecto de la relación e interdependencia entre el lugar, por un lado, y la existencia y creación de capital social, por otro, así como la influencia de ambos aspectos en la respuesta de la comunidad durante la emergencia. De igual modo, se espera poner de manifiesto la necesidad de considerar estos aspectos en las políticas de reconstrucción y en la planificación urbana, en el contexto de implementación de estrategias de reducción del riesgo de desastres. OEURE

\section{Referencias bibliográficas}

Comfort, L. K. (1999). Shared Risk: Complex systems in seismic response. Amsterdam: Pergamon.

Cruz Roja (2010). Social Media Grows Up - Red Cross emergency social data summit. En http://www. redcross.org/portal/site/en/menuitem.1a019a978f421296e81ec89e43181aa0/?vgnextoi $\mathrm{d}=$ fa532b019666a210VgnVCM10000089f0870aRCRD

Cutter, S. L., Boruff, B. J. \& Shirley, B. L. (2003). Social vulnerability to environmental hazards. Social Science Quarterly, 84(2), 242-261. doi: 10.1111/1540-6237.8402002

Cutter, S., Barnes, L., Berry, M., Burton, C., Evans, E., Tate, E. \& Webb, J. (2008a). A place-based model for understanding community resilience to natural disasters. Global Environmental Change, 18, 598-606. http://www.sciencedirect.com/science/article/pii/S0959378008000666

Cutter, S., Barnes, L., Berry, M., Burton, C., Evans, E., Tate, E. \& Webb, J. (2008b). Community and regional resilience: Perspectives from hazards, disasters and emergency management. CARRI Research Report 1. Oak Ridge, Tennessee: Community \& Regional Resilience Initiative. En http://www.resilientus.org/library/FINAL_CUTTER_9-25-08_1223482309.pdf

Durán, M. (2006). Plan Regulador San Pedro de la Paz. Etapa 1: Diagnóstico Urbano Comunal. Ministerio de Vivienda y Urbanismo. Concepción: Secretaría Regional Ministerial (Seremi) de Vivienda y Urbanismo, Región del Biobío.

Dynes, R. (2002). The importance of social capital in disaster response. Preliminary Paper No 327. Newark, DE: University of Delaware, Disaster Research Center. En http://dspace.udel. edu:8080/dspace/bitstream/handle/19716/292/PP\%20327.pdf ?sequence=1

EM-DAT (Emergency Events Database) (2012).Disaster profiles. http://www.emdat.be/disaster-profiles Estrategia Internacional para la Reducción de los Desastres (EIRD, Organización de las Naciones Unidas). (2005). Marco de Acción de Hyogo para 2005-2015: Aumento de la resiliencia de las naciones y las comunidades ante los desastres. Revisión de medio término 2010-2011 [Traducción provisoria]. En http://www.unisdr.org/files/18197_provisionalspanishversionmidtermrev.pdf 
Folke, C., Carpenter, S., Elmqvist, T., Gunderson, L., Holling, C. S. \& Walker, B. (2002). Resilience and sustainable development: building adaptive capacity in a world of transformations. Ambio, 31(5), 437- 40. En http://era-mx.org/biblio/Resilience.pdf

Folke, C., Hahn, T., Olsson, P. \& Norberg, J. (2005) Adaptive governance of socio-ecological systems. Annual Review of Environment and Resources, 30, 441-473. doi: 10.1146/annurev. energy.30.050504.144511

Franck, S. \& Pérez, L. (2009). Más que una suma de casas. La Unidad Vecinal Villa San Pedro de Coronel. Revista INVI, 27(64), 127-152. doi: 10.4067/S0718-83582009000300005

Ganor, M. \& Ben-Lavy, J. (2003). Community resilience: Lessons derived from Gilo under fire. Jour nal of Jewish Communal Service, 79(2-3) (Winter/Spring), 105-108. En http://d1lj5119p3qzy9.cloudfront.net/handle/10207/bitstreams/16280.pdf

Godschalk, D. R. (2003). Urban hazard mitigation: Creating resilient cities. Natural Hazards Review, 4(3), 136-143. doi: 10.1061/(ASCE)1527-6988(2003)4:3(136)

González-Muzzio, C. (2010). Exploring community resilience: The social-urban aftermath of the Biobio Earthquake. Tesis de magíster, Departamento de Geografía, University College London, Londres, Reino Unido. En http://es.scribd.com/doc/99101979/Gonzalez-Muzzio2010-Exploring-Community-Resilience

Greene, M. \& Mora, R. (2008). Dimensiones espaciales de la seguridad residencial: Flujos de movimiento y campos visuales. Revista INVI, 23(64), 143-166. En http://revistainvi.uchile.cl/ index.php/INVI/article/view/450/421

Inside Facebook (2010). After earthquake, Chilean users find friends and family on Facebook while world shares sympathies. En http://www.insidefacebook.com/2010/03/02/chilean-usersfinding-family-on-facebook-world-shares-sympathies/

Instituto Nacional de Estadísticas (INE). (2002). Censo de Población y Vivienda. Santiago: Autor. En http:// www.ine.cl/canales/chile_estadistico/censos_poblacion_vivienda/censo_pobl_vivi.php

Instituto Nacional de Estadísticas (INE). (2005). Chile hacia el 2050. Proyecciones de población. Santiago: Autor.

Mileti, D. S. (1999). Disasters by design: A reassessment of natural hazards in the United States. Washington, D.C.: Joseph Henry Press.

Ministerio de Vivienda y Urbanismo (Minvu), Chile. (2010, 29 de marzo). Chile Unido Reconstruye Mejor. Programa de Reconstrucción Nacional en Vivienda. Santiago: Autor. En http://www. superacionpobreza.cl/biblioteca-archivos/programa_reconstruccion.pdf

Norris F. H., Stevens, S. P., Pfefferbaum, B., Wyche, K. \& Pfefferbaum, R. L. (2008). Community resilience as a metaphor, theory, set of capacities, and strategy for disaster readiness. American Journal of Community Psychology, 41(1-2), 127-150.

Pérez, L. \& Salinas, E. (2007). Crecimiento urbano y globalización: Transformaciones del Área Metropolitana de Concepción, Chile, 1992-2002. Scripta Nova. Revista Electrónica de Geografía y Ciencias Sociales, 11(251). En http://www.ub.es/geocrit/sn/sn-251.htm

Quarantelli, E. L. (2004). Emergent behaviors and groups in the crisis time periods of disasters. Preliminary Paper $\mathrm{N}^{\circ}$ 206. Newark, DE: University of Delaware, Disaster Research Center. En http://dspace.udel.edu:8080/dspace/handle/19716/591

Quarantelli, E. L. (2007). The myth and the realities: keeping the "looting" myth in perspective. Natural Hazards Observer, 31(4), 2-3. En http://www.colorado.edu/hazards/o/archives $/ 2007 / \mathrm{mar} 07 / \mathrm{mar} 07 . \mathrm{pdf}$ 
Rose, A. (2004). Defining and measuring economic resilience to earthquakes. En Research Progress and Accomplishments, 2003-2004 (pp. 41-54) [Online]. Buffalo, NY: Multidisciplinary Center for Earthquake Engineering Research (MCEER), State University of New York at Buffalo. En https://mceer.buffalo.edu/publications/resaccom/04-sp01/04_rose.pdf

Sanzana, M. (2010). Desastre natural y acción colectiva de los sectores populares en Chile: los saqueos en Concepción tras el 27/F. Observatorio Social de América Latina (OSAL), 11(28), 145157. En http://biblioteca.clacso.edu.ar/ar/libros/osal/osal28/osal28.pdf

Smith, P. \& Romero, H. (2009). Efectos del crecimiento urbano del Área Metropolitana de Concepción sobre los humedales Rocuant-Andalién, Los Batros y Lenga. Revista de Geografía Norte Grande, 43, 81-93. doi: 10.4067/S0718-34022009000200005

Subsecretaría de Desarrollo Regional y Administrativo (Subdere). (2010). Ficha Comunal. San Pedro de la Paz. Sistema Nacional de Información Municipal (Sinim). En http://www.sinim.gov. cl/assets/fcomunal/ficha_comunal.php. Para el año 2009, en http://www.sinim.cl/ficha_ comunal/fcomunal.php?id_muni=08108\&ano=2009\&periodo=A

Sutton, J., Palen, L. \& Shklovski, I. (2008). Backchannels on the front lines: Emergent uses of social media in the 2007 Southern California wildfires. En F. Fiedrich \& B. van de Walle (Eds.), Proceedings of the 5th International ISCRAM Conference, Washington D.C. En http://jeannettesutton.com/uploads/BackchannelsISCRAM08.pdf

The United Nations Office for Disaster Risk Reduction (UNISDR). (2012). Disaster statistics. En http://www.unisdr.org/we/inform/disaster-statistics

Tierney, K. (2003). Conceptualizing and measuring organizational and community resilience: Lessons from the emergency response following the September 11, 2001 attack on the World Trade Center. Preliminary Paper $N^{\circ} 329$. Newark, DE: University of Delaware, Disaster Research Center. En http://dspace.udel.edu:8080/dspace/handle/19716/735

Tierney, K. (2009). Disaster response: Research findings and their implications for resilience measures. CARRI Research Report 6. Oak Ridge, Tennessee: Community and Regional Resilience Initiative. En http://www.resilientus.org/library/Final_Tierney2_dpsbjs_1238179110. $\mathrm{pdf}$

Twigg, J. (2007). Characteristics of a disaster-resilient community: A guidance note. Version 1 (for field testing), for the DFID Disaster Risk Reduction Interagency Coordination Group. En https://practicalaction.org/docs/ia1/community-characteristics-en-lowres.pdf

U.S. Indian Ocean Tsunami Warning System (IOTWS). (2006). Concepts and practices of "resilience": A compilation from various secondary sources. US IOTWS Program Document No. 05-IOTWS-06. A Working paper prepared for Coastal Community Resilience (CCR) Program, by Atiq Kainan Ahmed. Prepared for U.S. Agency for International Development by IRG (International Resources Group)-Tetra Tech Joint Venture under Contract No. EPPI-02-04-00024-00. En http://pdf.usaid.gov/pdf_docs/PNADY190.pdf

Wallace, D. \& Wallace, R. (2008). Urban systems during disasters: factors for resilience. Ecology and Society, 13(1), art. 18. [Online]. En http://www.ecologyandsociety.org/vol13/iss1/art18/ 